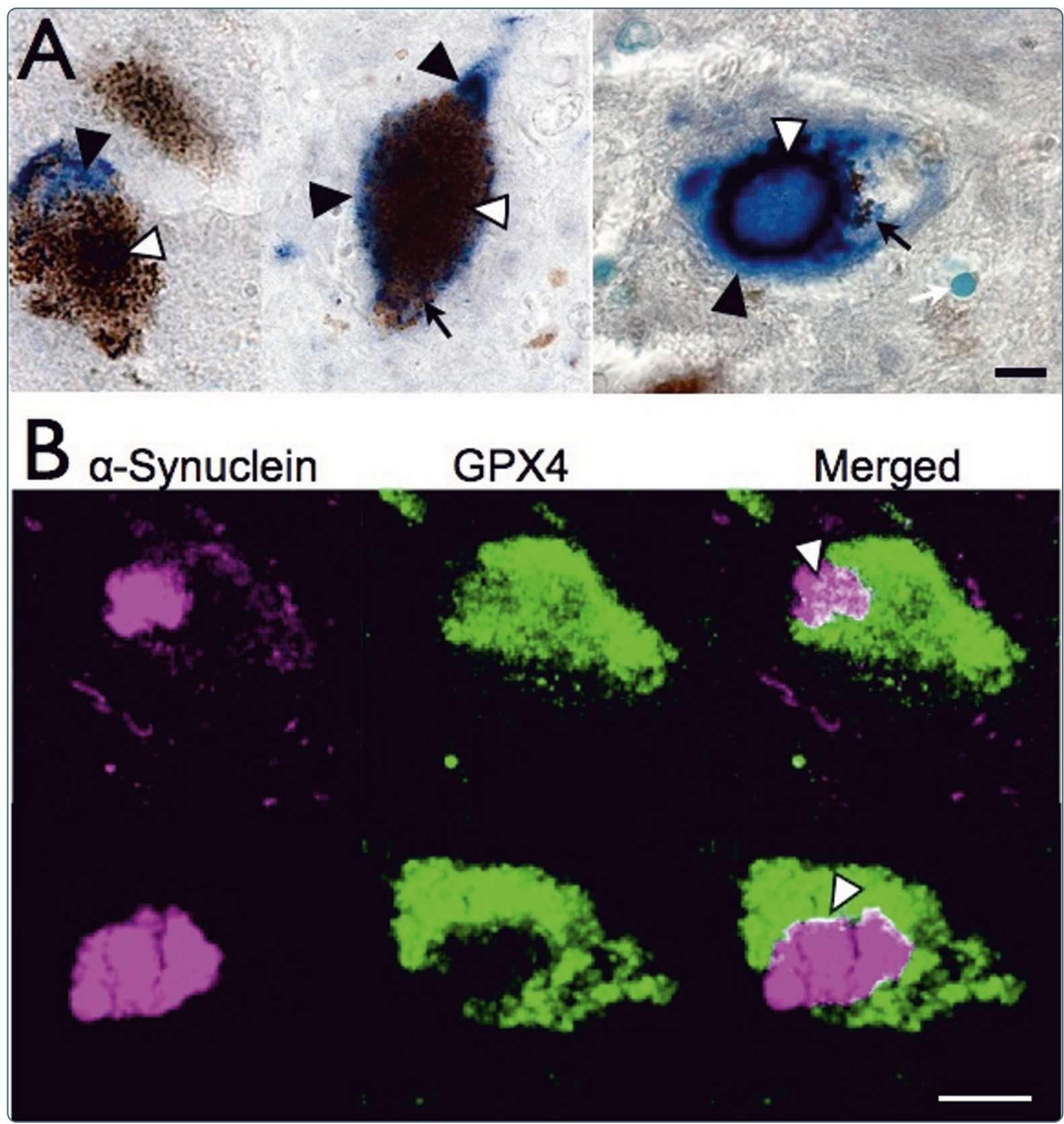

Glutathione Peroxidase 4 is associated with Neuromelanin in Substantia Nigra and Dystrophic Axons in Putamen of Parkinson's brain

Bellinger et al. 


\title{
Glutathione Peroxidase 4 is associated with Neuromelanin in Substantia Nigra and Dystrophic Axons in Putamen of Parkinson's brain
}

Frederick P Bellinger ${ }^{1 *}$, Miyoko T Bellinger ${ }^{1}$, Lucia A Seale ${ }^{1}$, Andrea S Takemoto ${ }^{1}$, Arjun V Raman ${ }^{1}$, Takanori Miki ${ }^{4}$, Amy B Manning-Boğ ${ }^{5}$, Marla J Berry ${ }^{1}$, Lon R White ${ }^{2}$, G Webster Ross ${ }^{2,3}$

\begin{abstract}
Background: Parkinson's disease is a neurodegenerative disorder characterized pathologically by the loss of nigrostriatal dopamine neurons that project from the substantia nigra in the midbrain to the putamen and caudate nuclei, leading to the clinical features of bradykinesia, rigidity, and rest tremor. Oxidative stress from oxidized dopamine and related compounds may contribute to the degeneration characteristic of this disease.

Results: To investigate a possible role of the phospholipid hydroperoxidase glutathione peroxidase 4 (GPX4) in protection from oxidative stress, we investigated GPX4 expression in postmortem human brain tissue from individuals with and without Parkinson's disease. In both control and Parkinson's samples, GPX4 was found in dopaminergic nigral neurons colocalized with neuromelanin. Overall GPX4 was significantly reduced in substantia nigra in Parkinson's vs. control subjects, but was increased relative to the cell density of surviving nigral cells. In putamen, GPX4 was concentrated within dystrophic dopaminergic axons in Parkinson's subjects, although overall levels of GPX4 were not significantly different compared to control putamen.
\end{abstract}

Conclusions: This study demonstrates an up-regulation of GPX4 in neurons of substantia nigra and association of this protein with dystrophic axons in striatum of Parkinson's brain, indicating a possible neuroprotective role. Additionally, our findings suggest this enzyme may contribute to the production of neuromelanin.

\section{Background}

Parkinson's disease (PD) is characterized pathologically by the development of cellular inclusions called Lewy bodies composed of aggregated proteins, primarily $\alpha$-synuclein (AS) as well as ubiquitin and other misfolded proteins. The presence of ubiquitin within these structures suggests a build-up of proteins that were directed towards proteolytic degradation. AS also accumulates in the dopaminergic projections from these neurons forming dystrophic axons. PD is associated with severe loss of dopamine (DA) neurons in the substantia nigra (SN) and their striatal terminals, leading to dopamine depletion and consequential motor impairments. Accumulation of oxidized biomolecules associated with degeneration of DA neurons indicates the

\footnotetext{
* Correspondence: fb@hawaii.edu

${ }^{1}$ Cell and Molecular Biology Department, John A. Burns School of Medicine, University of Hawaii, Honolulu, HI 96813 USA

Full list of author information is available at the end of the article
}

involvement of oxidative stress mechanisms [1], and the highly oxidizable aromatic structure of DA and its metabolites are thought to contribute to these processes [2].

Neurons in SN pigmented with neuromelanin are particularly sensitive to neurodegeneration in PD [3]. With aging, SN neurons in primates gradually transition from dopaminergic neurons, identified by the presence of the DA-synthesizing enzyme tyrosine hydroxylase $(\mathrm{TH})$, to dopaminergic neurons expressing neuromelanin and eventually to cells that do not synthesize dopamine (TH-negative) but have neuromelanin [4]. The formation is postulated to require hydroperoxidase activity. Peroxidase activity associated with neuromelanin has been demonstrated and is increased in postmortem PD brain [5]. However, a hydroperoxidase responsible for neuromelanin formation has not specifically been identified.

The glutathione peroxidases (GPX) are hydroperoxidases essential for maintaining redox balance in cells [6]. 
Most of the GPX enzymes are selenoproteins that contain the micronutrient selenium (Se) incorporated as the amino acid, selenocysteine [7]. Se deficiency results in impairments in neurological function [8], highlighting the importance in brain of proteins utilizing this essential trace element. GPXs have been implicated in PD as brain glutathione levels are decreased in early stages of PD [9]. As glutathione is a cofactor for the GPX enzymes, its loss results in decreased peroxidase activity [10]. Overexpression of GPX1 attenuates 6-OHDA induced toxicity to dopamine neurons in rodent models of PD [11,12]. A recent study showed that GPX1 is present in human microglia and may play a role in removing AS aggregates from neuron terminals [13].

Less is known about the possible role of GPX4 in PD. Whereas GPX1 reduces inorganic hydrogen peroxide, GPX4 is the major enzyme that reduces membrane lipid peroxides [7]. Recent studies indicate the importance of the phospholipid hydroperoxidase GPX4 in the brain. Genetic deletion of GPX4 is embryonic lethal $[14,15]$, and conditional deletion of GPX4 in brain results in severe neurodegeneration [16], indicating that the role of GPX4 in lipid hydroperoxide removal is essential for cell viability. Translation of the GPX4 protein is regulated by DJ-1, a protein implicated in a recessive form of early-onset PD [17]. Oxidation of DJ-1 is associated with an increase in cerebral cortex of GPX4 in subjects with sporadic Parkinson's disease [18]. However, as expression of GPX4 has not been investigated in the brain regions most affected in $\mathrm{PD}$, i.e. the substantia nigra $(\mathrm{SN})$ and basal ganglia, it is unclear if this upregulation is related to the etiology of PD or secondary to PD pathology. Lastly, the phospholipid hydroperoxidase function of GPX4 makes it a candidate enzyme for neuromelanin formation.

Here we evaluate the pattern of expression of GPX4 in the nigrostriatal pathway and whether expression is altered in PD as a possible response to oxidative stress. We report that GPX4 is co-localized with neuromelanin in SN dopamine neurons, decreased in PD midbrain, and associated with dystrophic axons in PD putamen.

\section{Results}

We examined GPX4 in postmortem brain of 12 subjects that had been clinically diagnosed with PD, as well as 11 subjects without clinical or postmortem pathological features of PD. Tissue was obtained from the HonoluluAsia Aging Study (HAAS). Data for all subjects are summarized in Table 1 . There were no significant differences in age or post-mortem interval (PMI) between control and PD subjects. However, data obtained at autopsy demonstrated that the density of cells in SN was reduced in PD subjects to about a third of controls. The degeneration was most severe in lateral ventral SN,
Table 1 Study Subjects

\begin{tabular}{rcc}
\hline & Control $(\boldsymbol{n}=\mathbf{1 1})$ & PD $(\boldsymbol{n}=\mathbf{1 2})$ \\
\hline Age Range (YR): & $76.6-91.7$ & $79.6-92.9$ \\
Age at Death (YR): & $84.0 \pm 1.3$ & $84.2 \pm 1.0$ \\
Post-Mortem Interval (HR): & $17.01 \pm 2.7$ & $9.57 \pm 4.1$ \\
Median (and Range) Braak stage: & $0(0)$ & $5.5(5-6)$ \\
Total SN Cell Density: & $21.7 \pm 1.9$ & $8.2 \pm 1.4^{\dagger}$ \\
Lateral-Ventral SN Cell Density: & $27.6 \pm 3.3$ & $5.2 \pm 1.6^{\dagger}$ \\
\hline
\end{tabular}

$\dagger$ indicates significantly different from controls $(P<0.0001)$.

HAAS subject information obtained at autopsy. Methods published previously [19].

All decedents were American-born males of Japanese ancestry living in Hawaii. Data are mean \pm SEM unless indicated otherwise.

where the cell density in PD subjects was approximately a fifth of control subjects [19].

\section{GPX4 in SN}

We tested GPX4 antibody specificity by western blot. As shown in Figure 1A, the antibody reacted with a $22 \mathrm{kD}$ band in protein from human brain and from SH-SY5Y human neuroblastoma cells. This immunoreactivity was blocked by preabsorbing the antibody with the recombinant GPX4 antigen. However, the antibody did not recognize protein in extracts from the mouse N2A neuroblastoma cell line, and reacted with additional bands in mouse cortex protein. Thus the antibody appeared to

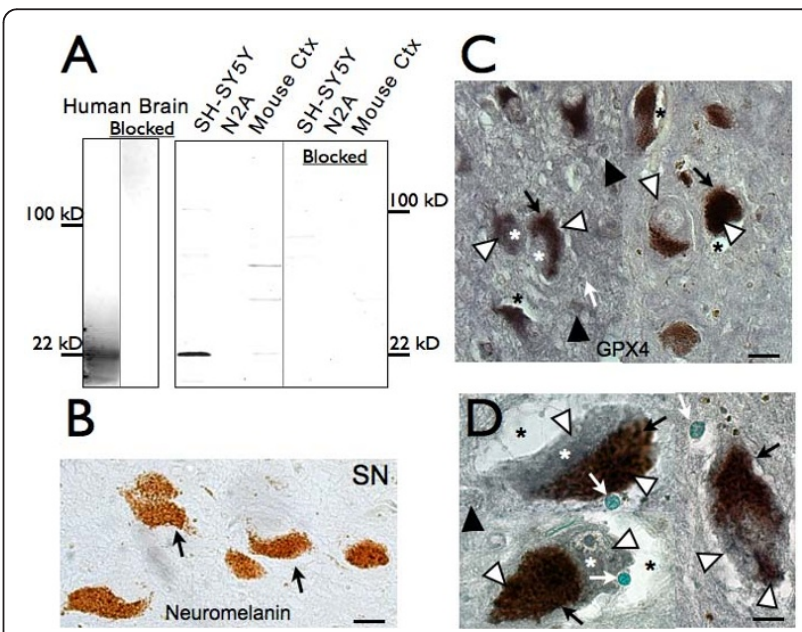

Figure 1 GPX4 is associated with neuromelanin in SN neurons. GPX4 colocalizes with neuromelanin in SN of control brain. A. Western blot showing antibody specificity in human brain (left) and human and mouse neuroblastoma cells and mouse cortex (right). For "blocked" controls, antibody was preabsorbed with original recombinant antigen. B. Immunohistochemistry negative control with primary antibody omitted. Brown pigment is endogenous neuromelanin (black arrows). C. Robust GPX4 expression (dark grey $\mathrm{Ni}$-DAB, white arrowheads) is associated with neuromelanin (brown, black arrows) and in the cytoplasm $\left(^{*}\right)$. D. Enlarged cell images, counterstained with methyl green to show nuclei of these neurons (white arrows). Scale bars: B, C, $20 \mu \mathrm{m}, \mathrm{D}, 10 \mu \mathrm{m}$. 
be very specific for GPX4 in human brain but not in mouse cells and tissue.

We examined subcellular localization of GPX4 in SN in control brain using immunohistochemistry. GPX4 was mostly colocalized with neuromelanin in SN neurons (Figure $1 \mathrm{C}$ ). SN neuromelanin can be clearly seen when primary antibody is omitted (Figure 1B). GPX4 immunolabeling with 3, 3-diaminobenzidine hydrochloride (DAB) containing $\mathrm{NiCl}_{2}$, in order to contrast with neuromelanin, coincided mostly within neuromelaninpositive neurons, and surprisingly was mostly associated with intracellular neuromelanin (Figure 1C, D). Weaker labeling of other cytoplasmic areas in these neurons is also present (shown by white asterisks), but some cytoplasmic regions were void of GPX4 immunoreactivity and appeared as empty space (black asterisks). Co-localization of GPX4 with neuromelanin was observed in midbrain sections from all 11 control and 12 PD subjects, although neuromelanin was notably reduced in SN of PD midbrain, presumably due to loss of neuromelanincontaining neurons. GPX4 labeling was also present at low levels in tissue surrounding neurons, in smaller glia cells (black arrowheads) and in midbrain white matter fibers (not shown).

We used spectral imaging to compare GPX4 immunoreactivity in SN cells with presence or absence of neuromelanin. Figure 2A depicts a group of TH-positive cells that are negative for neuromelanin. GPX4 immunoreactivity is largely absent in these cells, although it can be seen in surrounding glia and white matter. Figure $2 \mathrm{~B}$ shows a group of $\mathrm{TH}$ - and neuromelanin-positive neurons. GPX4 immunoreactivity was visible in most TH-positive cells expressing neuromelanin, although expression levels varied from cell to cell. Figure $2 \mathrm{C}$ shows a group of $\mathrm{TH}$-negative, neuromelanin-positive cells. GPX4 labeling was strongest within these cells. These findings suggest GPX4 expression increases in SN neurons with the development of neuromelanin and continues to increase as expression of $\mathrm{TH}$ is lost.

\section{GPX4 in PD SN}

We examined GPX4 immunoreactivity in SN of PD and control brain and its relation to Lewy bodies using an antibody to alpha synuclein. We found GPX4 associated with AS-positive Lewy bodies (Figure 3A), which were often found within or bordering cellular regions of GPX4/neuromelanin. This is in agreement with the association of GPX4 with neuromelanin, as Lewy bodies have some association with neurons that have high neuromelanin content [3]. Confocal sections though tissue with a thickness of $0.9 \mu \mathrm{m}$ showed that GPX4 labeling present within Lewy bodies (3B, above), although generally less than in surrounding cytoplasm and typically confined to the perimeter of the inclusions (3B, below).

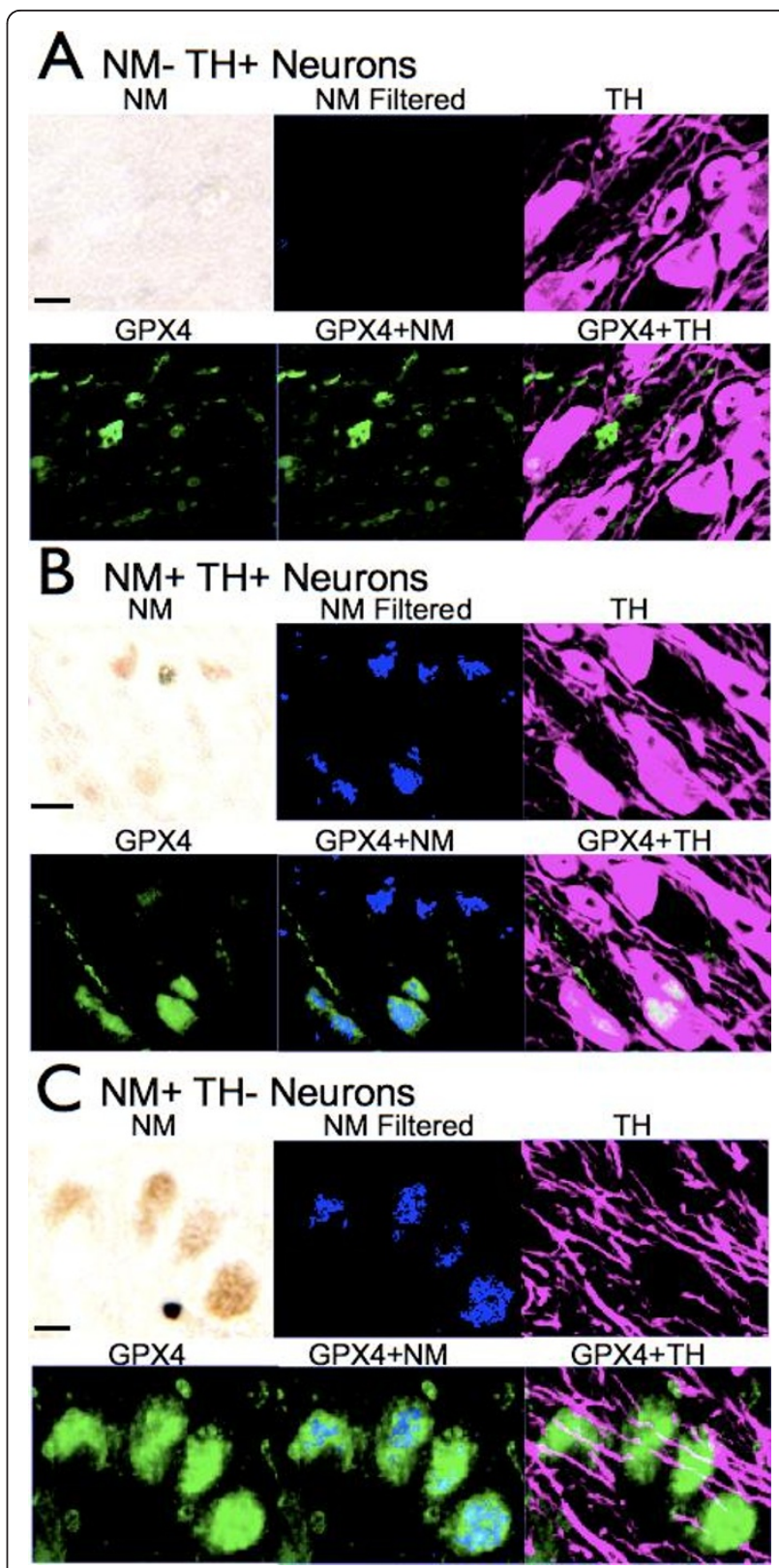

Figure 2 GPX4 is found primarily in Neuromelanin-expressing neurons of SN. GPX4 immunoreactivity is present in most cells expressing neuromelanin but is absent in many cells expressing $\mathrm{TH}$ but not neuromelanin. A. Example of cells expressing TH but not neuromelanin. Light microscope images (above left) were filtered for neuromelanin (blue, above center) to compare with $\mathrm{TH}$ immunoreactivity (magenta, above right) and GPX4 immunoreactivity (green, below left). GPX4 images combined with neuromelanin (below, middle) and TH immunoreactivity (below, right) are also shown for comparison. GPX4 is present in surrounding glia but absent in $\mathrm{TH}$-expressing neurons. B. Examples of $\mathrm{TH}$ and neuromelanin expressing neurons. GPX4 is present in most cells with neuromelanin. C. GPX4 immunoreactivity is prominent in neurons containing neuromelanin but no longer expressing TH. (Note: as images were not taken with confocal microscopy, color changes only show only co-localization of area and not intracellular co-localization). Scale bars: $20 \mu \mathrm{m}$. 


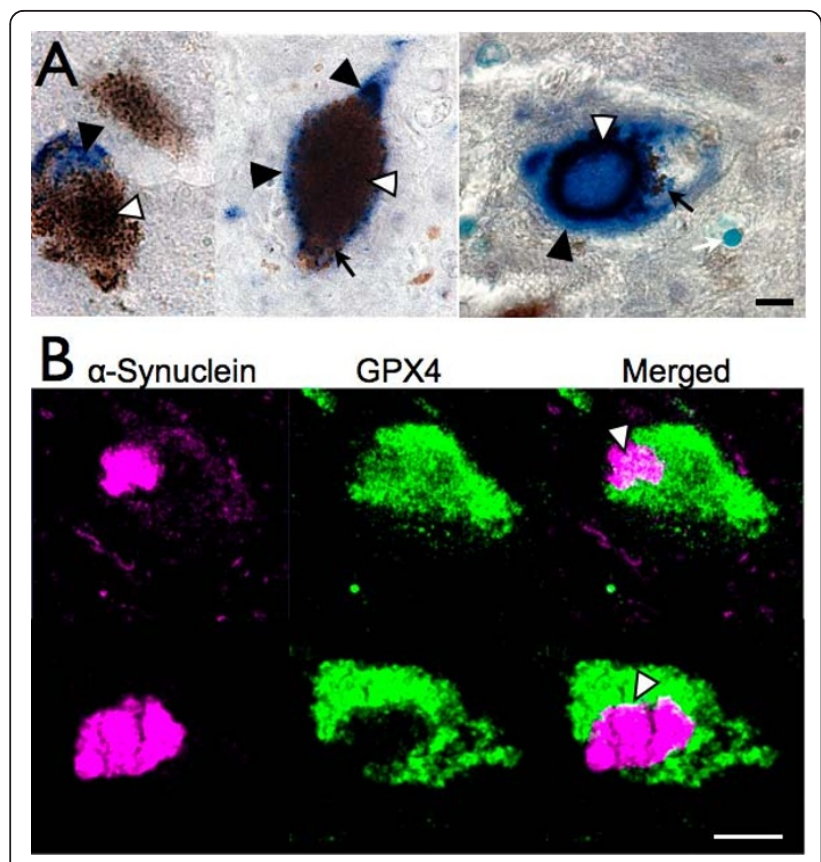

Figure 3 GPX4 in nigral inclusions of PD brain. A. GPX4 expression (dark grey Ni-DAB, white arrowheads) coincides with ASpositive Lewy bodies (blue BCIP, marked by black arrowheads) in SN. Black arrows indicate neuromelanin. B. Confocal microscope images of two cells showing relationship of GPX4 (green) to AS (magenta). The above example shows some co-localization of GPX4 with AS within a Lewy body, while the below example shows GPX4 only around the Lewy body perimeter. Scale bars: A, $20 \mu \mathrm{m}, \mathrm{B}, 10 \mu \mathrm{m}$.

We investigated possible changes in GPX4 in PD brain by measuring area of immunoreactivity using unbiased stereology. Using a Cavalieri probe, we determined that GPX4 was significantly decreased in SN of PD brain compared with control SN (Figure 4A, B). The total volume fraction of GPX4 immunoreactivity in this region was reduced by nearly one third in $\mathrm{PD}$ brain, from $0.036 \pm 0.004$ in control SN $(n=11)$ to $0.024 \pm$ 0.003 in PD SN $(\mathrm{n}=12)$.

The reduction in total GPX4 immunoreactivity could be due to the severe cell loss in this region. In order to determine if loss of GPX4 could be explained by cell death, we investigated the volume of GPX4 as a function of cell density. We found that GPX4 immunoreactivity is actually increased relative to the cell density of surviving SN neurons (Figure 4C). GPX4 volume density is increased from $0.0018 \pm 0.0002$ in controls $(\mathrm{n}=$ $11)$ to $0.0038 \pm 0.0007$ in $P D(n=12)(P=0.0192)$, indicating that GPX4 is either upregulated in surviving neurons or that a greater percentage of cells not expressing GPX4 are lost compared to cells with GPX4. This suggests a neuroprotective role for GPX4 within these neurons.

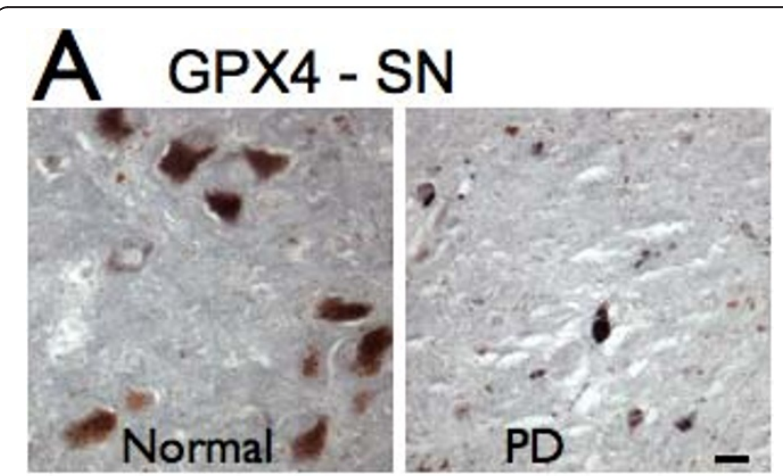

\section{B SN-Volume Fraction}

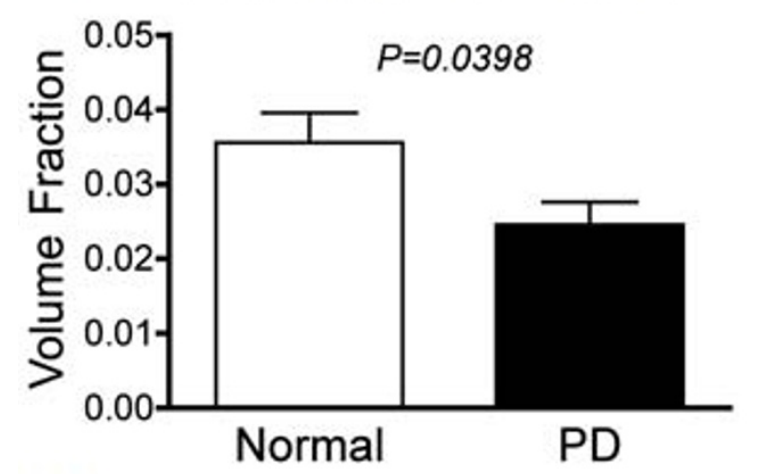

\section{SN-Volume Density}

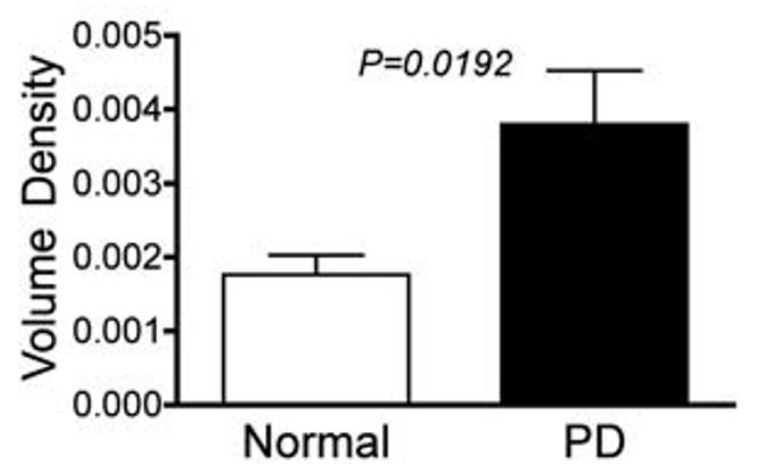

Figure 4 GPX4 is reduced overall but increased relative to cell density in PD SN. A. GPX4 (dark grey) is visibly reduced in SN of PD subjects ( $n=12$ ) compared to controls $(n=11)$. B. Total immunoreactivity of GPX4 is significantly reduced in SN of PD subjects compared to controls ( $P=0.0398$, Student's t-test). C. GPX4 immunoreactivity is increased relative to cell density $(P=0.0192$, Student's t-test). Scale bars: $20 \mu \mathrm{m}$.

\section{GPX4 in Putamen}

In putamen, GPX4 is associated with small cell bodies reminiscent of small stellate neurons or glia (Figure 5A). GPX4 immunoreactivity was not significantly different between normal and PD putamen (Figure 5B). The area 


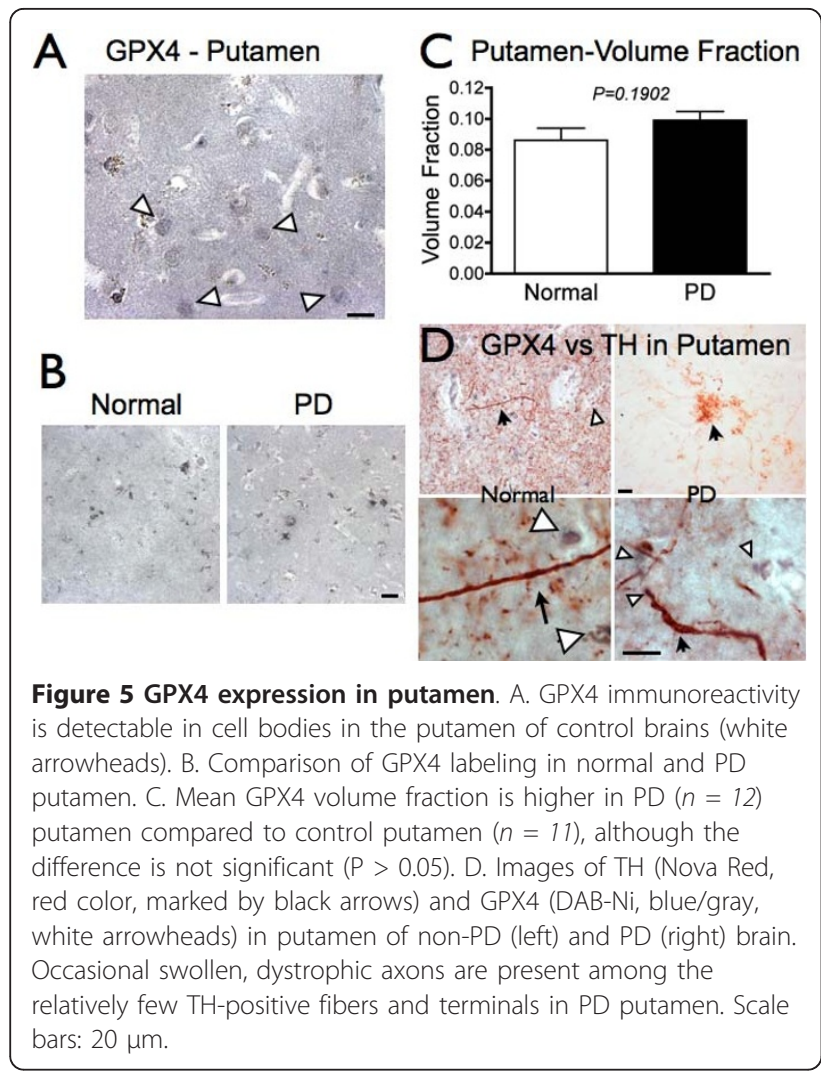

of GPX4 immunoreactivity was slightly increased in PD putamen compared to controls, but the increase was not significant (Figure 5C). As PD-associated neurodegeneration is specific to DA axons from SN and not cell bodies in putamen, this finding supports a loss of GPX that is specific within DA neurons.

To determine if GPX4 was found in DA terminals, we used double-labeling immunohistochemistry with GPX4 and an antibody to tyrosine hydroxylase (TH). In nonPD putamen sections, GPX4 immunoreactivity (blue/ gray) was generally not associated with $\mathrm{TH}$-positive (red) fibers in control brain (Figure 5C, left), although weaker levels of GPX4 immunoreactivity are possible. Instead, GPX4 was mostly associated with smaller cells within putamen that are likely to be stellate neurons or glial cells. PD putamen contained relatively few TH-positive fibers and terminals, and some of the TH-positive axons were varicose and truncated (5D, right). Some GPX4 immunoreactivity was associated with the dystrophic axons in PD putamen (Figure 5D, lower right), in contrast with the lack of GPX4 found in $\mathrm{TH}$-positive terminals in non-PD subjects.

We used confocal microscopy to further examine the relationship between GPX4 and TH in putamen axons (Figure 6). As described for light microscopy, we found little colocalization of GPX4 with TH in putamen samples from non-PD subjects. However, we found GPX4

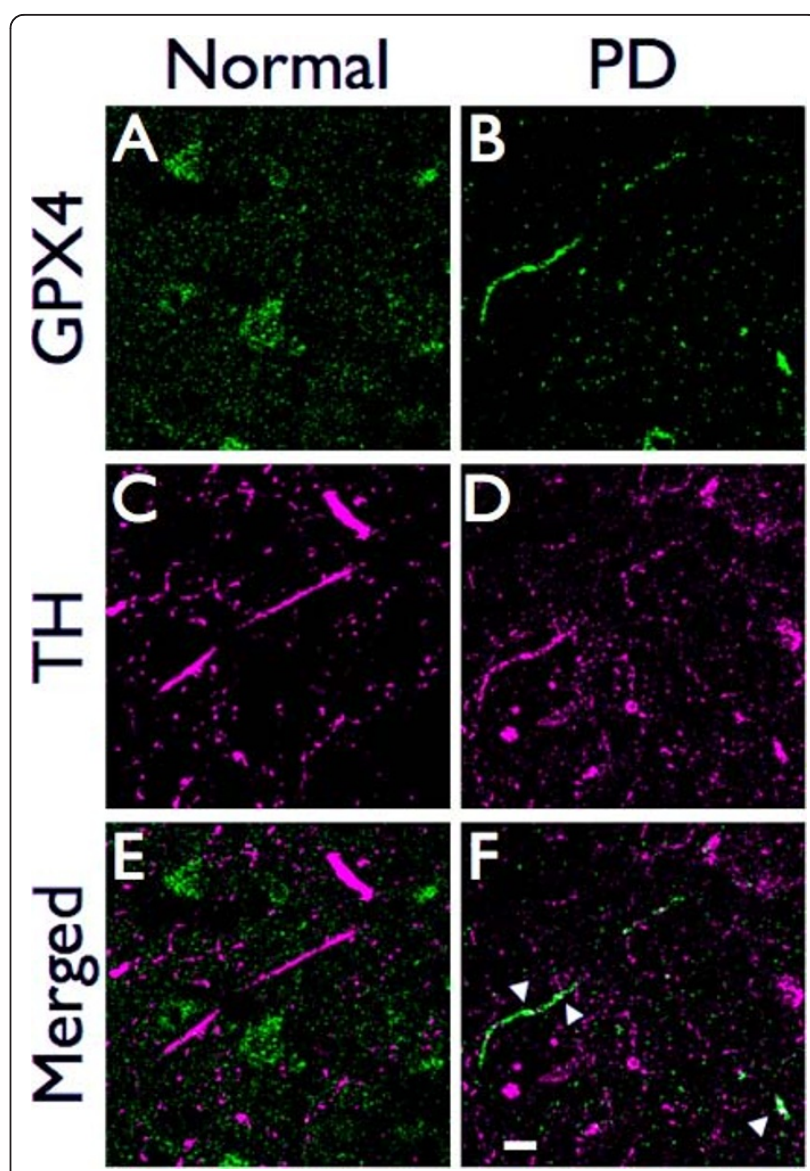

Figure 6 GPX4 is associated with dystrophic axons in PD putamen. Confocal images of GPX4 (blue) and TH (magenta) in nonPD (and PD brain (F, H, J). GPX4 has little association with TH in control brain sections, but colocalizes with $\mathrm{TH}$ in dystrophic axons in PD brain (shown by white color, white arrowheads). Scale bar: $10 \mu \mathrm{m}$.

concentrated in dystrophic axons and colocalization of GPX4 with TH in axonal varicosities (shown by white arrowheads). This suggests an upregulation of GPX4 in degenerating dopaminergic terminals.

Altogether, these findings indicate changes in GPX4 in $\mathrm{SN}$ and putamen that coincide with PD pathology, suggesting a role for GPX4 in the development of this disorder.

\section{Discussion}

In this study, we have determined that altered GPX4 levels and distribution are associated with pathological PD changes. Specifically, overall GPX4 is greatly reduced in the SN of PD vs. control subjects, but when cell loss is taken into account, GPX4 is increased relative to cell density. Interestingly, GPX4 immunoreactivity is associated with neuromelanin in SN and colocalizes with AS-positive nigral Lewy bodies and dystrophic TH-positive fibers in putamen. These findings indicate either an 
upregulation of GPX4 in response to PD pathology, or an increase in survival of cells expressing GPX4, and further suggest a neuroprotective role of GPX4 in PD pathology.

GPX4 has recently been shown to prevent $12 / 15$ lipoxygenase-dependent apoptotic cell death resulting from oxidative stress [16]. In the absence of GPX4, lipid peroxidation via 12/15-lipoxygenase leads to activation of the apoptosis-inducing factor (AIF) to induce cell death. Thus GPX4 is an important factor to prevent neuronal death from the increased lipid peroxidation found in PD neurons. However, GPX4 may be less effective in PD brain as glutathione levels are reduced early in the disorder [9]. This could lead to overproduction of GPX4 without the benefit of reduced oxidative stress and lead to its association with pathological structures.

Colocalization of GPX4 with neuromelanin is particularly suggestive of a role for GPX4 in aging of DA neurons. As GPX4 is a lipid hydroperoxidase [20], colocalization with neuromelanin suggests GPX4 could be a hydroperoxidase contributing to the synthesis of neuromelanin. The function of neuromelanin is currently unknown, but it is relevant that the substance accumulates in the SN of aging primates [4,21]. Neuromelanin is specific to catecholaminergic neurons of higher mammals, and SN neuromelanin is comprised of repeats of quinone structures which are likely formed from oxidized dopamine as well as dopamine synthesis or breakdown structures such as L-DOPA and DOPAC [21-23]. In a previous proteomics study, GPX4 was identified as a protein associated with neuromelanin in human brain [24]. Buildup of neuromelanin may be toxic to cells [25], although there is some evidence that it has a protective role in these neurons [26]. The colocalization of GPX4 with neuromelanin could indicate a response to oxidized lipids associated with neuromelanin. As neuromelanin is made up of lipids, protein and fatty acids, GPX4 may associate with neuromelanin in order to reduce the associated oxidized molecules. Conversely, an association of GPX4 with neuromelanin may result in a buildup of oxidized glutathione, further depleting the active pool of glutathione in PD.

The association of GPX4 with TH-positive degenerating axons in putamen suggests an up-regulation of GPX4 at the level of the putamen. A buildup of oxidized dopamine in axons could possibly induce expression of GPX4 to mitigate oxidative stress. An increase in GPX4, specific to dystrophic axons, could explain the apparent increase in GPX4 in putamen, which may be masked by a lack of change in cell bodies of neurons intrinsic to putamen. Furthermore, it seems possible that the changes in GPX4 in dystrophic neurons could be related to the colocalization of GPX4 with neuromelanin. Up-regulated GPX4 may accumulate in cell bodies, or may be transported from fibers as axons retract. The increase in GPX4 in soma may catalyze formation of neuromelanin from increased dopamine quinone in these neurons. Thus GPX4 may have an important role in the development of PD pathology.

\section{Conclusions}

We have developed a model of the possible competing reactions of GPX4 in reduction of lipids and production of neuromelanin, depicted in Figure 7. GPX4 uses glutathione as a substrate to reduce oxidized lipids (above) [15]. However, a build-up of oxidized forms of DA-related compounds such as DA-quinones may drive the synthesis of neuromelanin from these compounds, a process that may compete with reduction of lipids (below) [21-23]. Depletion of glutathione and upregulation of GPX4 peroxidase activity in response to oxidative stress may further drive the production of neuromelanin

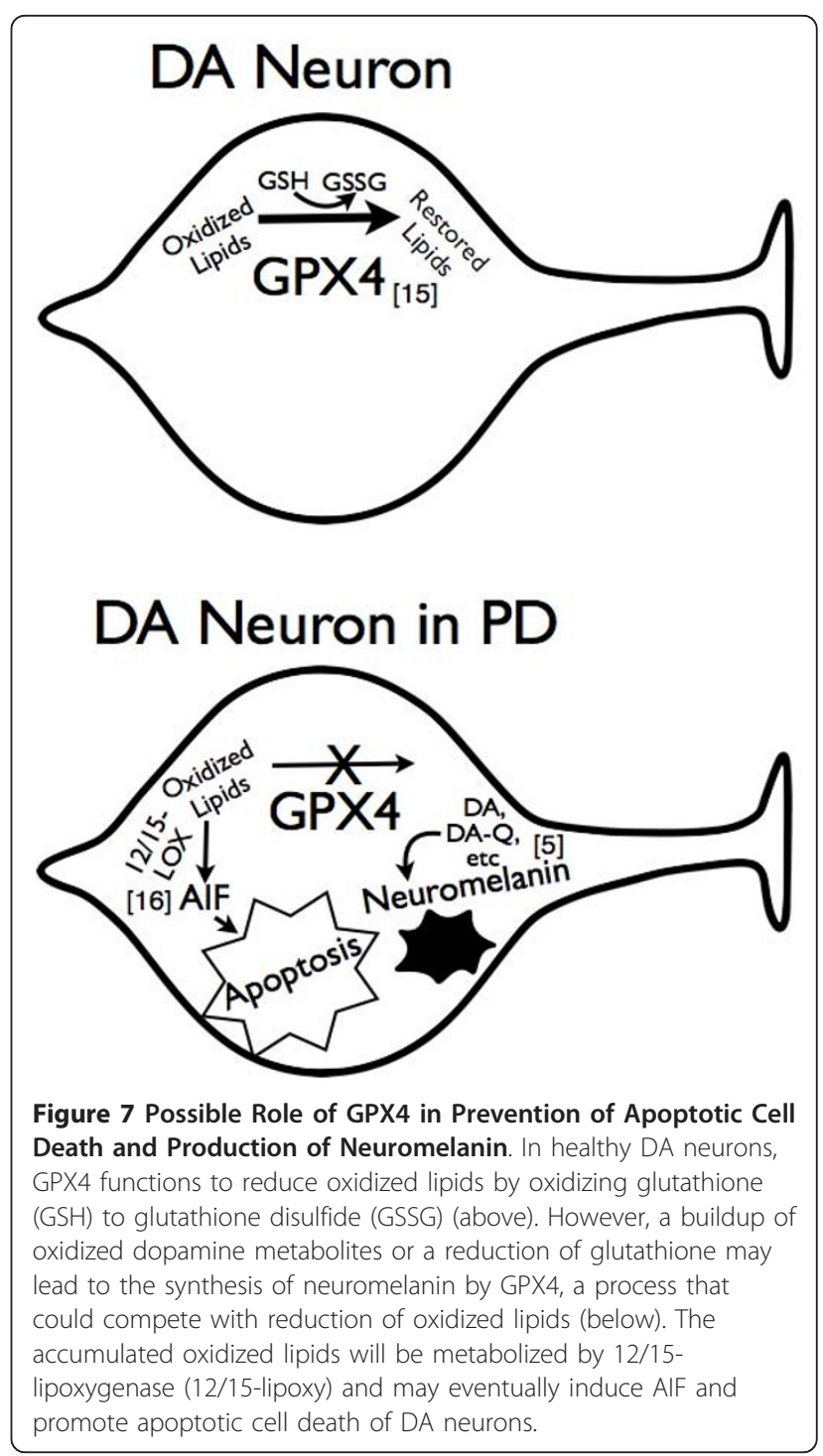


and inhibit reduction of lipids [5]. Accumulated oxidized lipids are metabolized by 12/15-lipoxygenase, and metabolite products activate AIF to promote apoptotic cell death [16]. Thus under pathological conditions such as extreme levels of oxidation, the important protective properties of GPX4 may be redirected towards neuromelanin production, and this process may contribute to the loss of DA neurons in PD. Overall, our findings indicate an important neuroprotective role for GPX4 in neurons of the nigrostriatal pathway, and also suggest a role in neuromelanin synthesis associated with primate aging.

\section{Methods}

\section{Subjects}

Formalin-fixed human brain tissue was provided by the Honolulu-Asia Aging Study (HAAS), an ongoing longitudinal epidemiological study that has monitored the health and lifestyle of a cohort of Japanese-American men born between 1900 and 1919 and residing on Oahu, Hawaii [27]. Sections $(10 \mu \mathrm{m})$ of SN and putamen from 12 subjects clinically diagnosed with PD and having marked pathological features including Lewy bodies and degeneration of dopaminergic terminals and cell bodies were examined. Sections from 11 age-matched subjects that had no symptoms of PD during life and no PD-associated neuropathology were used as controls in this study.

\section{Western blot}

Post-mortem tissue from human occipital cortex was homogenized by sonication in $10 \mathrm{mM}$ Tris/1 mM EDTA/protease inhibitor cocktail (1:1000; Sigma, St. Louis, MO), centrifuged at $1,000 \times \mathrm{g}$ for $10 \mathrm{~min}$, and protein concentration in the supernatant was measured using Pierce BCA kit (Rockford, IL). Proteins were separated by SDS-PAGE and transferred to nitrocellulose. Protein was extracted from SH-SY5Y cells, N2A cells and mouse cortex using CellLytic buffer (Sigma) per manufacturer's instructions, separated by electrophoresis and blotted to PVDF membranes. Blots were blocked with Odyssey blocking buffer (LiCore Biosciences) for $1 \mathrm{hr}$ and then incubated in GPX4 antibody (AbFrontier) diluted 1:2000. To confirm antibody specificity, antibody blocked with $50 \mu \mathrm{g} / \mathrm{ml}$ recombinant GPX4 antigen (AbFrontier) was also used. After washing with PBS containing $0.05 \%$ tween-20 (PBST), membranes were treated with secondary antibodies labeled with infrared fluorophores (LiCore Biosciences). After further washes in PBST, blots were imaged with the Odyssey infrared imaging system (LiCore Biosciences).

\section{Immunolabeling}

Immunolabeling was performed as described previously [28]. Samples were de-paraffinized and antigens unmasked by heating in a pressure cooker to $95^{\circ} \mathrm{C}$ and 15 psi for 20 min in Trilogy alkaline solution with EDTA (Cell Marque), followed by $3 \mathrm{~min}$ in $90 \%$ formic acid. Samples were then blocked in PBS with 5\% serum, species matched to secondary antibody. Tissue was incubated in rabbit polyclonal anti-GPX4 (1:800, AbFrontier) overnight at $4^{\circ} \mathrm{C}$ in $3 \%$ serum. After washes, sections were incubated in biotinylated secondary antibody followed by $A B C^{\mathrm{TM}}$ reagent. HRP signals were developed with 3, 3-diaminobenzidine hydrochloride (DAB, Vector Labs), with or without the addition of nickel chloride to darken color as per manufacturer's instructions.

\section{Double Immunolabeling}

Following first primary antibody, tissue was subsequently blocked in 5\% normal horse serum, followed with separate blocking steps in streptavidin and biotin solutions (from $A B C$ kit) five minutes each before second primary antibody reaction. Additional primary antibodies used were anti- $\alpha$-synuclein (AS) (1:1000, Chemicon or 1:50, Abcam) and anti-tyrosine hydroxylase (TH, 1:8000, Sigma). Combinations of HRP-labeled secondary antibodies detected with $\mathrm{DAB}, \mathrm{DAB}$ containing nickel chloride (DAB-Ni) or Nova Red reactions (Vector Laboratories), or alkaline phosphatase (AP) detected with BCIP reactions, were used to maximize contrast between the different antibodies.

\section{Fluorescent Immunolabeling}

Depariffinization, antigen unmasking and primary antibody labeling were performed as described above. After washes, tissue was incubated in secondary antibodies conjugated to Alexa 488 and Alexa 546. Endogenous fluorescence was reduced by treating with an autofluorescence eliminator reagent (Chemicon).

\section{Spectral Imaging and Confocal Microscopy}

Bright light and fluorescent images of midbrain tissue samples were imaged using an Olympus microscope equipped with the Nuance multispectral imaging system (Cambridge Research and Instrumentation, Inc). After obtaining spectral libraries for bright light images of unlabeled tissue and neuromelanin and fluorescent images of fluorophores and background autofluorescence, brightfield and fluorescent images were "unmixed" into individual signal components (i.e. neuromelanin or fluorescent probes) that were pseudocolored for comparison.

Confocal images were collected with a Zeiss LSM Pascal laser confocal microscope and analyzed with ImageJ software.

\section{Stereology}

Volume-density of immunolabeling was determined with a Cavalieri probe using Stereologer software 
(Stereology Resource Center). First, the region (SN or putamen) was outlined using $5 \times$ magnification, and a computer-generated array of systematic-random loci were then visited and observed under 40x. A Cavalieri probe was placed over $50 \%$ of the image with an array of points $(+)$, and the fraction of points contacting immunolabeled cells were used to estimate the area fraction of immunolabeling at that location. The total area fraction of immunolabeling was estimated as the average area fraction of all systematic-randomly chosen sites. According to the Delesse principle, area fraction on random sections is equivalent to the volume fraction [29].

\section{List of Abbreviations}

AS: alpha synuclein; DA: dopamine; DAB: 3-diaminobenzidine hydrochloride; GPX: glutathione peroxidase; GPX1: glutathione peroxidase 1; GPX4: glutathione peroxidase 4 (phospholipid hydroperoxidase); HAAS: Honolulu-Asia Aging Study; PD: Parkinson's disease; PMl: post-mortem interval; Se: selenium; SN: substantia nigra; TH: tyrosine hydroxylase.

\section{Acknowledgements}

The authors thank Kristen Ewell for tissue sectioning, Yanling Lin and Chrislyn Andres for technical assistance, and Chris Zarow and Linda Chang for helpful comments and suggestions. Supported by: US Department of the Army grant DAMD17-98-1-8621 and the Office of Research and Development, Medical Research Service, Department of Veterans Affairs (GWR), Hawaii Community Foundation Grant 20071373 (FPB), NIH RO1 NS40302 (MJB), NIH R24 DA027318 (MJB/FPB), NIH U01 AG019349 (LRW), and $\mathrm{NIH}$ G12 RR003061 which supports the JABSOM histology/imaging core facility. The information contained in this paper does not necessarily reflect the position or the policy of the government, and no official endorsement should be inferred.

\section{Author details}

${ }^{1}$ Cell and Molecular Biology Department, John A. Burns School of Medicine, University of Hawaii, Honolulu, HI 96813 USA. ${ }^{2}$ Kuakini Medical Center, Honolulu, HI 96817 USA. ${ }^{3}$ Veterans Affairs Pacific Islands Health Care System in Honolulu, Honolulu, HI. ${ }^{4}$ Department of Anatomy, University of Kagawa, Saiwai, Takamatsu, 760-8521 Japan. ${ }^{5}$ Center for Health Sciences, SRI International, Menlo Park, CA 94025 USA.

\section{Authors' contributions}

FPB, GWR, LRW, and MJB designed the studies. ABM-B, AVR and TM aided with design detail and contributed essential interpretations of findings. MTB, AST and FPB performed the immunohistochemistry and FPB and ABM-B performed western blots. FPB, LAS and AVR performed microscopy and imaging. AST and LAS performed stereology. FPB wrote the paper with the assistance of all authors, who have read and approved the final manuscript

\section{Competing interests}

The authors declare that they have no competing interests.

Received: 30 September 2010 Accepted: 21 January 2011

Published: 21 January 2011

\section{References}

1. Adams JD Jr, Chang ML, Klaidman L: Parkinson's disease-redox mechanisms. Curr Med Chem 2001, 8:809-814.

2. Chinta SJ, Andersen JK: Dopaminergic neurons. Int J Biochem Cell Biol 2005, 37:942-946.

3. Halliday GM, Ophof A, Broe M, Jensen PH, Kettle E, Fedorow $H$, Cartwright MI, Griffiths FM, Shepherd CE, Double KL: Alpha-synuclein redistributes to neuromelanin lipid in the substantia nigra early in Parkinson's disease. Brain 2005, 128:2654-2664.
4. McCormack AL, Di Monte DA, Delfani K, Irwin I, DeLanney LE, Langston WJ, Janson AM: Aging of the nigrostriatal system in the squirrel monkey. J Comp Neurol 2004, 471:387-395.

5. De luliis A, Burlina AP, Boschetto R, Zambenedetti P, Arslan P, Galzigna L: Increased dopamine peroxidation in postmortem Parkinsonian brain. Biochim Biophys Acta 2002, 1573:63-67.

6. Arthur JR: The glutathione peroxidases. Cell Mol Life Sci 2000 , 57:1825-1835.

7. Bellinger FP, Raman AV, Reeves MA, Berry MJ: Regulation and function of selenoproteins in human disease. Biochem J 2009, 422:11-22.

8. Chen J, Berry MJ: Selenium and selenoproteins in the brain and brain diseases. J Neurochem 2003, 86:1-12.

9. Zeevalk GD, Razmpour R, Bernard LP: Glutathione and Parkinson's disease: is this the elephant in the room? Biomed Pharmacother 2008,62:236-249.

10. Ambani LM, Van Woert MH, Murphy S: Brain peroxidase and catalase in Parkinson disease. Arch Neurol 1975, 32:114-118.

11. Bensadoun JC, Mirochnitchenko O, Inouye M, Aebischer P, Zurn AD: Attenuation of 6-OHDA-induced neurotoxicity in glutathione peroxidase transgenic mice. Eur J Neurosci 1998, 10:3231-3236.

12. Ridet JL, Bensadoun JC, Deglon N, Aebischer P, Zurn AD: Lentivirusmediated expression of glutathione peroxidase: neuroprotection in murine models of Parkinson's disease. Neurobiol Dis 2006, 21:29-34.

13. Power $J \mathrm{H}$, Blumbergs $\mathrm{PC}$ : Cellular glutathione peroxidase in human brain: cellular distribution, and its potential role in the degradation of Lewy bodies in Parkinson's disease and dementia with Lewy bodies. Acta Neuropathol 2009, 117:63-73.

14. Imai H, Hirao F, Sakamoto $T$, Sekine K, Mizukura Y, Saito M, Kitamoto T, Hayasaka M, Hanaoka K, Nakagawa Y: Early embryonic lethality caused by targeted disruption of the mouse PHGPx gene. Biochem Biophys Res Commun 2003, 305:278-286.

15. Yant $L$, Ran $Q$, Rao L, Van Remmen H, Shibatani T, Belter JG, Motta L, Richardson A, Prolla TA: The selenoprotein GPX4 is essential for mouse development and protects from radiation and oxidative damage insults. Free Radic Biol Med 2003, 34:496-502.

16. Seiler A, Schneider M, Forster H, Roth S, Wirth EK, Culmsee C, Plesnila N, Kremmer $\mathrm{E}$, Radmark $\mathrm{O}$, Wurst W, et al: Glutathione peroxidase 4 senses and translates oxidative stress into 12/15-lipoxygenase dependent- and AlF-mediated cell death. Cell Metab 2008, 8:237-248.

17. van der Brug MP, Blackinton J, Chandran J, Hao LY, Lal A, MazanMamczarz K, Martindale J, Xie C, Ahmad R, Thomas KJ, et al: RNA binding activity of the recessive parkinsonism protein DJ-1 supports involvement in multiple cellular pathways. Proc Natl Acad Sci USA 2008, 105:10244-10249.

18. Blackinton J, Kumaran R, van der Brug MP, Ahmad R, Olson L, Galter D, Lees A, Bandopadhyay $R$, Cookson MR: Post-transcriptional regulation of mRNA associated with DJ-1 in sporadic Parkinson disease. Neurosci Lett 2009, 452:8-11.

19. Ross GW, Petrovitch H, Abbott RD, Nelson J, Markesbery W, Davis D, Hardman J, Launer L, Masaki K, Tanner CM, White LR: Parkinsonian signs and substantia nigra neuron density in decendents elders without PD. Ann Neurol 2004, 56:532-539.

20. Brigelius-Flohe R: Glutathione peroxidases and redox-regulated transcription factors. Biol Chem 2006, 387:1329-1335.

21. Sulzer D, Bogulavsky J, Larsen KE, Behr G, Karatekin E, Kleinman MH, Turro N, Krantz D, Edwards RH, Greene LA, Zecca L: Neuromelanin biosynthesis is driven by excess cytosolic catecholamines not accumulated by synaptic vesicles. Proc Natl Acad Sci USA 2000, 97:11869-11874

22. Smythies J, De luliis A, Zanatta L, Galzigna L: The biochemical basis of Parkinson's disease: the role of catecholamine o-quinones: a reviewdiscussion. Neurotox Res 2002, 4:77-81.

23. Smythies J, Galzigna L: The oxidative metabolism of catecholamines in the brain: a review. Biochim Biophys Acta 1998, 1380:159-162.

24. Tribl F, Gerlach M, Marcus K, Asan E, Tatschner T, Arzberger T, Meyer HE, Bringmann G, Riederer P: "Subcellular proteomics" of neuromelanin granules isolated from the human brain. Mol Cell Proteomics 2005, 4:945-957.

25. Hastings TG: The role of dopamine oxidation in mitochondrial dysfunction: implications for Parkinson's disease. J Bioenerg Biomembr 2009, 41:469-472. 
26. Double KL, Ben-Shachar D, Youdim MB, Zecca L, Riederer P, Gerlach M: Influence of neuromelanin on oxidative pathways within the human substantia nigra. Neurotoxicol Teratol 2002, 24:621-628.

27. White L, Petrovitch H, Ross GW, Masaki KH, Abbott RD, Teng EL,

Rodriguez BL, Blanchette PL, Havlik RJ, Wergowske G, et al: Prevalence of dementia in older Japanese-American men in Hawaii: The Honolulu-Asia Aging Study. Jama 1996, 276:955-960.

28. Bellinger FP, He QP, Bellinger MT, Lin Y, Raman AV, White LR, Berry MJ: Association of selenoprotein $\mathrm{P}$ with Alzheimer's pathology in human cortex. J Alzheimers Dis 2008, 15:465-472.

29. Mouton PR, Long JM, Lei DL, Howard V, Jucker M, Calhoun ME, Ingram DK: Age and gender effects on microglia and astrocyte numbers in brains of mice. Brain Res 2002, 956:30-35.

doi:10.1186/1750-1326-6-8

Cite this article as: Bellinger et al:: Glutathione Peroxidase 4 is associated with Neuromelanin in Substantia Nigra and Dystrophic Axons in

Putamen of Parkinson's brain. Molecular Neurodegeneration 2011 6:8.

\section{Submit your next manuscript to BioMed Central} and take full advantage of:

- Convenient online submission

- Thorough peer review

- No space constraints or color figure charges

- Immediate publication on acceptance

- Inclusion in PubMed, CAS, Scopus and Google Scholar

- Research which is freely available for redistribution

Submit your manuscript at www.biomedcentral.com/submit
C Biomed Central 